\title{
Exact solutions for the Einstein-Gauss-Bonnet theory in five dimensions: Black holes, wormholes and spacetime horns
}

\author{
Gustavo Dotti ${ }^{1}$, Julio Oliva ${ }^{2,3}$, and Ricardo Troncoso ${ }^{3}$ \\ ${ }^{1}$ Facultad de Matemática, Astronomía y Física, Universidad Nacional de Córdoba, \\ Ciudad Universitaria, (5000) Córdoba, Argentina. \\ ${ }^{2}$ Departamento de Física, Universidad de Concepción, \\ Casilla, 160-C, Concepción, Chile. and \\ ${ }^{3}$ Centro de Estudios Científicos (CECS), Casilla 1469, Valdivia, Chile.
}

\begin{abstract}
An exhaustive classification of certain class of static solutions for the five-dimensional EinsteinGauss-Bonnet theory in vacuum is presented. The class of metrics under consideration is such that the spacelike section is a warped product of the real line with a nontrivial base manifold. It is shown that for generic values of the coupling constants the base manifold must be necessarily of constant curvature, and the solution reduces to the topological extension of the Boulware-Deser metric. It is also shown that the base manifold admits a wider class of geometries for the special case when the Gauss-Bonnet coupling is properly tuned in terms of the cosmological and Newton constants. This freedom in the metric at the boundary, which determines the base manifold, allows the existence of three main branches of geometries in the bulk. For negative cosmological constant, if the boundary metric is such that the base manifold is arbitrary, but fixed, the solution describes black holes whose horizon geometry inherits the metric of the base manifold. If the base manifold possesses a negative constant Ricci scalar, two different kinds of wormholes in vacuum are obtained. For base manifolds with vanishing Ricci scalar, a different class of solutions appears resembling "spacetime horns". There is also a special case for which, if the base manifold is of constant curvature, due to certain class of degeneration of the field equations, the metric admits an arbitrary redshift function. For wormholes and spacetime horns, there are regions for which the gravitational and centrifugal forces point towards the same direction. All these solutions have finite Euclidean action, which reduces to the free energy in the case of black holes, and vanishes in the other cases. The mass is also obtained from a surface integral.
\end{abstract}

Electronic addresses: gdotti-at-famaf.unc.edu.ar, juliooliva-at-cecs.cl, ratron-at-cecs.cl 


\section{Contents}

I. Introduction

II. Exact solutions and their classification

III. Geometrically well behaved solutions: Black holes, wormholes and spacetime horns

A. Case (ii.a): Black holes

B. Case (ii.b): Wormholes and spacetime horns

C. Geodesics around wormholes and spacetime horns

1. Radial geodesics

2. Gravitational vs. centrifugal forces

\section{Regularized Euclidean action}

A. Geometrically well-behaved solutions as extrema of the regularized action

B. Euclidean action for geometrically well-behaved solutions

\section{Mass from a surface integral}

\section{Discussion and comments}

\section{References}

\section{INTRODUCTION}

According to the basic principles of General Relativity, higher dimensional gravity is described by theories containing higher powers of the curvature [1]. In five dimensions, the most general theory leading to second order field equations for the metric is the so-called Einstein-Gauss-Bonnet theory, which contains quadratic powers of the curvature. The pure gravity action is given by

$$
I=\kappa \int d^{5} x \sqrt{g}\left(R-2 \Lambda+\alpha\left(R^{2}-4 R_{\mu \nu} R^{\mu \nu}+R_{\alpha \beta \gamma \delta} R^{\alpha \beta \gamma \delta}\right)\right),
$$

where $\kappa$ is related to the Newton constant, $\Lambda$ to the cosmological term, and $\alpha$ is the GaussBonnet coupling. For later convenience, it is useful to express the action (1) in terms of 
differential forms as

$$
I=\int \epsilon_{a b c d e}\left(\alpha_{2} R^{a b} R^{c d}+\alpha_{1} R^{a b} e^{c} e^{d}+\alpha_{0} e^{a} e^{b} e^{c} e^{d}\right) e^{e}
$$

where $R^{a b}=d \omega^{a b}+\omega_{f}^{a} \omega^{f b}$ is the curvature 2-form for the spin connection $\omega^{a b}=\omega_{\mu}^{a b} d x^{\mu}$, $e^{a}=e_{\mu}^{a} d x^{\mu}$ is the vielbein and the wedge product is understood ${ }^{1}$. For a metric connection with vanishing torsion, the field equations from (2) read

$$
\mathcal{E}_{a}:=\epsilon_{a b c d e}\left(\alpha_{2} R^{b c} R^{d e}+3 \alpha_{1} R^{b c} e^{d} e^{e}+5 \alpha_{0} e^{b} e^{c} e^{d} e^{e}\right)=0
$$

The kind of spacetimes we are interested in have static metrics of the form

$$
d s^{2}=-f^{2}(r) d t^{2}+\frac{d r^{2}}{g^{2}(r)}+r^{2} d \Sigma_{3}^{2},
$$

where $d \Sigma_{3}^{2}$ is the line element of a three-dimensional manifold $\Sigma_{3}$ that we call the "base manifold". Note that $\partial / \partial t$ is a timelike Killing vector field, orthogonal to 4-manifolds that are a warped product of $\mathbb{R}$ with the base manifold $\Sigma_{3}$.

If the Gauss-Bonnet coupling $\alpha_{2}$ vanishes, General Relativity with a cosmological constant is recovered. In this case the equations force the base manifold to be of constant curvature $\gamma$ (which can be normalized to $\gamma= \pm 1$ or zero) and ${ }^{2}$ [2]

$$
f^{2}=g^{2}=\gamma-\frac{\mu}{r^{2}}-\frac{5}{3} \frac{\alpha_{0}}{\alpha_{1}} r^{2}
$$

If $\gamma=1$, i.e., for $\Sigma_{3}=S^{3}$, the Schwarzschild-anti-de Sitter solution is recovered.

For spacetime dimensions higher than five, the equations of General Relativity do not impose the condition that the base manifold be of constant curvature. In fact, any Einstein base manifold is allowed [3]. For nonzero $\alpha_{2}$, however, the presence of the Gauss-Bonnet term restricts the geometry of an Einstein base manifold by imposing conditions on its Weyl tensor [7].

In this work we restrict ourselves to five dimensions without assuming any a priori condition on the base manifold in the ansatz (44). We show that in five dimensions, the presence of the Gauss-Bonnet term permits to relax the allowed geometries for the base manifold $\Sigma_{3}$, so that the whole structure of the five-dimensional metric turns out to be

\footnotetext{
${ }^{1}$ The relationship between the constants appearing in Eqs (10) and (2) is given by $\alpha=\frac{\alpha_{2}}{6 \alpha_{1}}, \Lambda=10 \frac{\alpha_{0}}{\alpha_{1}}$, $\kappa=-6 \alpha_{1}$.

${ }^{2}$ The four dimensional case was discussed previously in [4], [5], 6].
} 
sensitive to the geometry of the base manifold. More precisely, it is shown that solutions of the form (4) can be classified in the following way:

○ (i) Generic class: For generic coefficients, i.e., for arbitrary $\alpha_{0}, \alpha_{1}, \alpha_{2}$, the line element (4) solves the Einstein-Gauss-Bonnet field equations provided the base manifold $\Sigma_{3}$ is of constant curvature $\gamma$ (that we normalize to $\pm 1,0)$ and

$$
f^{2}=g^{2}(r)=\gamma+\frac{3}{2} \frac{\alpha_{1}}{\alpha_{2}} r^{2}\left[1 \pm \sqrt{\left(1-\frac{20}{9} \frac{\alpha_{2} \alpha_{0}}{\alpha_{1}^{2}}\right)+\frac{\mu}{r^{4}}}\right]
$$

where $\mu$ is an integration constant [8]. In the spherically symmetric case, (6) reduces to the well known Boulware-Deser solution [9].

○ (ii) Special class: In the special case where the Gauss-Bonnet coupling is given by

$$
\alpha_{2}=\frac{9}{20} \frac{\alpha_{1}^{2}}{\alpha_{0}}
$$

the theory possesses a unique maximally symmetric vacuum [10], and the Lagrangian can be written as a Chern-Simons form [11]. The solution set splits into three main branches according to the geometry of the base manifold $\Sigma_{3}$ :

- (ii.a) Black holes:

These are solutions of the form (41) with

$$
f^{2}=g^{2}=\sigma r^{2}-\mu, \quad \sigma:=\frac{10}{3} \frac{\alpha_{0}}{\alpha_{1}}
$$

( $\mu$ an integration constant). Their peculiarity is that with the above choice of $f$ and $g$, any (fixed) base manifold $\Sigma_{3}$ solves the field equations. Note that for negative cosmological constant $(\sigma>0)$ this solution describes a black hole [12], [13], which in the case of spherical symmetry, reduces to the one found in [9], [14].

- (ii.b1) Wormholes and spacetime horns: 
For base manifolds $\Sigma_{3}$ of constant nonvanishing Ricci scalar, $\tilde{R}=6 \gamma$, the metric (4) with

$$
\begin{aligned}
& f^{2}(r)=\left(\sqrt{\sigma} r+a \sqrt{\sigma r^{2}+\gamma}\right)^{2}, \\
& g^{2}(r)=\sigma r^{2}+\gamma
\end{aligned}
$$

( $a$ is an integration constant) is a solution of the field equations. In this case, there are three subbranches determined by $|a|>1,|a|<1$ or $|a|=1$. It is simple to show that, for negative cosmological constant $(\sigma>0)$ and $\gamma=-1$, the solution with $|a|<1$ corresponds to the wormhole in vacuum found in [15]. The solution with $|a|=1$ and $\gamma=-1$ corresponds to a brand new wormhole in vacuum (See Section III).

If the base manifold $\Sigma_{3}$ has vanishing Ricci scalar, i.e., $\tilde{R}=0$, it must be

$$
\begin{aligned}
& f^{2}(r)=\left(a \sqrt{\sigma} r+\frac{1}{\sqrt{\sigma} r}\right)^{2}, \\
& g^{2}(r)=\sigma r^{2}
\end{aligned}
$$

with $a$ an integration constant. If $\sigma>0$ and $a \geq 0$ this solution looks like a "spacetime horn". If the base manifold is not locally flat, there is a timelike naked singularity, but nevertheless the mass of the solution vanishes and the Euclidean continuation has a finite action (See Section IV).

\section{· (ii.b2) Degeneracy:}

If $\Sigma_{3}$ is of constant curvature, $\tilde{R}^{m n}=\gamma \tilde{e}^{m} \tilde{e}^{n}$, and $g^{2}$ given by Eq. (10), then the function $f^{2}(r)$ is left undetermined by the field equations.

The organization of the paper is the following: in Section II we solve the field equations and arrive at the classification outlined above, Section III is devoted to describing the geometry of the solutions of the special class, including some curious issues regarding the nontrivial behavior of geodesics around wormholes and spacetime horns. The Euclidean continuation of these solutions and the proof of the finiteness of their Euclidean action is worked out in Section IV. The mass of these solutions is computed from surface integrals in Section V. Section VI is devoted to a discussion of our results, and some further comments. 


\section{EXACT SOLUTIONS AND THEIR CLASSIFICATION}

In this Section we solve the field equations and arrive at the classification outlined in Section II. This is done in two steps. We first solve the constraint equation $\mathcal{E}_{0}=0$, and find two different cases: (i) a solution which is valid for any Einstein-Gauss-Bonnet theory, (ii) a solution that applies only to those theories satisfying (7).

In a second step we solve the remaining field equations and complete the classification of the solution set.

The vielbein for the metric (4) is chosen as

$$
e^{0}=f d t, e^{1}=g^{-1} d r, e^{m}=r \tilde{e}^{m},
$$

where $\tilde{e}^{m}$ stands for the vielbein on the base manifold, so that the indices $m, n, p \ldots$ run along $\Sigma_{3}$. The constraint equation $\mathcal{E}_{0}=0$ then acquires the form

$$
B_{0}(r) \tilde{R}+6 A_{0}(r)=0
$$

where $\tilde{R}$ is the Ricci scalar of the base manifold, and

$$
\begin{aligned}
& A_{0}=20 \alpha_{0} r^{4}-3 \alpha_{1} r\left(g^{2} r^{2}\right)^{\prime}+\alpha_{2}\left(g^{4}\right)^{\prime} r \\
& B_{0}=2 r\left[3 \alpha_{1} r-\alpha_{2}\left(g^{2}\right)^{\prime}\right]
\end{aligned}
$$

Since $\tilde{R}$ depends only on the base manifold coordinates, Eq.(14) implies that

$$
A_{0}(r)=-\gamma B_{0}(r)
$$

where $\gamma$ is a constant. Hence, the constraint reduces to

$$
\left\{\begin{array}{l}
B_{0}(r)(\tilde{R}-6 \gamma)=0 \\
A_{0}(r)=-\gamma B_{0}(r)
\end{array}\right.
$$

and implies that either

(i) the base manifold is of constant Ricci scalar $\tilde{R}=6 \gamma$, or

(ii) $B_{0}=0$. 
In case (i) the solution to (17) is

$$
g^{2}(r)=\gamma+\frac{3}{2} \frac{\alpha_{1}}{\alpha_{2}} r^{2}\left[1 \pm \sqrt{\left(1-\frac{20}{9} \frac{\alpha_{2} \alpha_{0}}{\alpha_{1}^{2}}\right)+\frac{\mu}{r^{4}}}\right],
$$

( $\mu$ is an integration constant). Since this solution holds for generic values of $\alpha_{0}, \alpha_{1}$ and $\alpha_{2}$ we call case (i) the generic branch.

Case (ii), on the other hand, implies $A_{0}=B_{0}=0$ (see equation (17)), and this system admits a solution only if the constants of the theory are tuned as in (7), the solution being

$$
g^{2}=\sigma r^{2}-\mu, \quad \sigma:=\frac{10}{3} \frac{\alpha_{0}}{\alpha_{1}}
$$

Note that in case (ii) the constraint equation does not impose any condition on the base manifold.

The radial equation $\mathcal{E}_{1}=0$, combined with the constraint in the form $e^{0} \mathcal{E}_{0}-e^{1} \mathcal{E}_{1}=0$ reduces to

$$
\left(B_{0}(r)-B_{1}(r)\right) \tilde{R}+6\left(A_{0}(r)-A_{1}(r)\right)=0
$$

where

$$
\begin{aligned}
& A_{1}(r)=2 r\left[10 \alpha_{0} r^{3}-3 \alpha_{1} g^{2} r-3 \alpha_{1} g^{2} \frac{f^{\prime}}{f} r^{2}+2 \alpha_{2} \frac{f^{\prime}}{f} g^{4}\right], \\
& B_{1}(r)=2 r\left[3 \alpha_{1} r-2 \alpha_{2} g^{2} \frac{f^{\prime}}{f}\right] .
\end{aligned}
$$

Finally, the three "angular" field equations $\mathcal{E}_{m}=0$ are equivalent to the following three equations

$$
B(r) \tilde{R}^{m n}+A(r) \tilde{e}^{m} \tilde{e}^{n}=0
$$

where

$A(r):=60 \alpha_{0} r^{4}+\frac{\alpha_{2} r^{2}}{f}\left(3\left(g^{4}\right)^{\prime} f^{\prime}+4 g^{4} f^{\prime \prime}\right)-3 \alpha_{1} r^{2}\left(2\left(g^{2} r\right)^{\prime}+4 g^{2} \frac{f^{\prime}}{f} r+\left(g^{2}\right)^{\prime} \frac{f^{\prime}}{f} r^{2}+2 g^{2} \frac{f^{\prime \prime}}{f} r^{2}\right)$

and

$$
B:=2 r^{2}\left[3 \alpha_{1}-\alpha_{2}\left(\left(g^{2}\right)^{\prime} \frac{f^{\prime}}{f}+2 g^{2} \frac{f^{\prime \prime}}{f}\right)\right]
$$

In what follows we solve the field equations (21) and (22), starting from the generic case (i), i.e., base manifolds with a constant Ricci scalar $\tilde{R}=6 \gamma$, and $g^{2}$ given by (19). 
- Radial and angular equations, Generic case (i): The radial field equation $\mathcal{E}_{1}=0$ allows to find the explicit form of the function $f^{2}(r)$, whereas the components of the field equations along the base manifold restricts its geometry to be of constant curvature. This is seen as follows:

Since in case (i) the base manifold has $\tilde{R}=6 \gamma$, where $\gamma$ is a constant, Eq.(21) reads

$$
\left(B_{0}(r)-B_{1}(r)\right) \gamma+\left(A_{0}(r)-A_{1}(r)\right)=0
$$

its only solution being $f^{2}=C g^{2}$, where the constant $C$ can be absorbed into a time rescaling. Thus, in the generic case (i), the solution to the field equations $\mathcal{E}_{0}=\mathcal{E}_{1}=0$ for the ansatz (41) is $f^{2}=g^{2}$ given in (19)

The angular equations (22) imply

$$
A(r)=-\lambda B(r)
$$

for some constant $\lambda$, and then (22) is equivalent to

$$
\left\{\begin{array}{l}
B(r)\left(\tilde{R}^{m n}-\lambda \tilde{e}^{m} \tilde{e}^{n}\right)=0, \\
A(r)=-\lambda B(r)
\end{array}\right.
$$

Since $B(r) \neq 0$ for $f^{2}=g^{2}$ given by (19), the base manifold must necessarily be of constant curvature, i.e., the metric of $\Sigma_{3}$ satisfies $\tilde{R}^{m n}=\lambda \tilde{e}^{m} \tilde{e}^{n}$, and, since $\tilde{R}=6 \gamma$, it must be $\lambda=\gamma$. This takes care of the first of equations (27). The second one adds nothing new since

$$
A(r)+\gamma B(r)=0
$$

is trivially satisfied because for $f=g$,

$$
r^{-2}[A(r)+\gamma B(r)]=r^{-1}\left[A_{0}(r)+\gamma B_{0}(r)\right]^{\prime},
$$

and $g$ satisfies (17). This concludes the classification of case (i).

- Radial and angular equations, Special case (ii): From the constraint equation $\mathcal{E}_{0}=0$, one knows that in this case, the Gauss-Bonnet coefficient is fixed as in Eq. (77), and the metric function $g^{2}$ is given by Eq. (201). 
The radial field equation (21) now reads

$$
\left(\left[\mu-\sigma r^{2}\right] \frac{f^{\prime}}{f}+\sigma r\right)(\tilde{R}+6 \mu)=0,
$$

which is solved either by

(ii.a) Having the first factor in (30) vanish, or by

(ii.b) Requiring the Ricci scalar of $\Sigma_{3}$ to be $\tilde{R}=-6 \mu$.

After a time re-scaling, the solution in case (ii.a), is $f^{2}=g^{2}$, (given in Eq. (201)).

No restriction on $\Sigma_{3}$ is imposed in this case.

Case (ii.b), on the other hand, is solved by requiring $\tilde{R}=-6 \mu$, so that the scalar curvature of the base manifold is related to the constant of integration in (20). Note that, in this case, the metric function $f^{2}$ is left undetermined by the system $\mathcal{E}_{0}=\mathcal{E}_{1}=0$.

The remaining fields equations, $\mathcal{E}_{m}=0$, can be written as

$$
\left(\sigma-\sigma r \frac{f^{\prime}}{f}-\left(\sigma r^{2}-\mu\right) \frac{f^{\prime \prime}}{f}\right)\left(\tilde{R}^{m n}+\mu \tilde{e}^{m} \tilde{e}^{n}\right)=0 .
$$

For case (ii.a), the first factor of Eq. (31) vanishes, and the geometry of base manifold $\Sigma_{3}$ is left unrestricted. We have a solution of the full set of field equations of the special theories (17) given by (4) with $f^{2}=g^{2}$ of Eq. (201), and an arbitrary base manifold $\Sigma_{3}$. In case (ii.b), Eq.(31) can be solved in two different ways:

(ii.b1) Choosing $f$ such that the first factor vanishes.

(ii.b2) Requiring the base manifold to be of constant curvature $-\mu$, i.e., $\tilde{R}^{m n}=-\mu \tilde{e}^{m} \tilde{e}^{n}$.

Case (ii.b2) leaves the redshift function $f^{2}$ completely undetermined.

Case (ii.b1) opens new interesting possibilities. The vanishing of the first factor of Eq. (31) gives a differential equation for the redshift function, whose general solution, after a time rescaling, reads

$$
f^{2}(r)=\left\{\begin{array}{c}
\left(\sqrt{\sigma} r+a \sqrt{\sigma r^{2}-\mu}\right)^{2}: \mu \neq 0 \\
\left(a \sqrt{\sigma} r+\frac{1}{\sqrt{\sigma} r}\right)^{2} \\
: \mu=0
\end{array},\right.
$$

where $a$ is an integration constant. $\Sigma_{3}$ is not a constant curvature manifold, although it has constant Ricci scalar $\tilde{R}=-6 \mu$. Note that we do not loose generality if we set $-\mu$ equal to $\gamma= \pm 1,0$.

For $\gamma \neq 0$ there are three distinct cases, namely $|a|>1,|a|<1$ or $|a|=1$, with substantially different qualitative features. It is simple to show that, for negative cosmological 
constant $(\sigma>0)$, the solution with $\gamma=-1$ and $|a|<1$ corresponds to the wormhole in vacuum found in [15], whereas that with $|a|=1$ corresponds to a brand new wormhole in vacuum (See Section III).

On the other hand, if $\gamma=0$ (base manifold with vanishing Ricci scalar), for negative cosmological constant and nonnegative $a$, the metric (44) describes a spacetime that looks like a "spacetime horn". We will see in the next section that if the base manifold is not locally

flat, there is a timelike naked singularity. Yet, the mass of the solution vanishes and the Euclidean continuation has a finite action (See Section [IV]).

This concludes our classification of solutions. Since case (i) has been extensively discussed in the literature, we devote the following sections to a discussion of the novel solutions (ii)a and (ii)b1/b2.

\section{GEOMETRICALLY WELL BEHAVED SOLUTIONS: BLACK HOLES, WORMHOLES AND SPACETIME HORNS}

In this Section we study the solutions for the special case found above.

One can see that, when they describe black holes and wormholes, as $r$ goes to infinity the spacetime metric approaches that of a spacetime of constant curvature $-\sigma$, with different kinds of base manifolds. This is also the case for spacetime horns, provided $a \neq 0$ (See Sec. III. B). It is simple to verify by inspection that for $\sigma \leq 0$, the solutions within the special case are geometrically ill-behaved in general. Hence, hereafter we restrict our considerations to the case $l^{2}:=\sigma^{-1}>0$, where $l$ is the anti-de Sitter (AdS) radius.

\section{A. Case (ii.a): Black holes}

According to the classification presented in the previous section, fixing an arbitrary base manifold $\Sigma_{3}$, the metric

$$
d s^{2}=-\left(\frac{r^{2}}{l^{2}}-\mu\right) d t^{2}+\frac{d r^{2}}{\left(\frac{r^{2}}{l^{2}}-\mu\right)}+r^{2} d \Sigma_{3}^{2} .
$$

solves the full set of Einstein Gauss Bonnet equations for the special theories (7). The integration constant $\mu$ is related to the mass, which is explicitly computed from a surface integral in Section V] For $\mu>0$, the metric (33) describes a black hole whose horizon is 
located at $r=r_{+}:=\sqrt{\mu} l$. Requiring the Euclidean continuation to be smooth, the black hole temperature can be obtained from the Euclidean time period, which is given by

$$
\beta=\frac{1}{T}=\frac{2 \pi l^{2}}{r_{+}} .
$$

For later purposes it is useful to express the Euclidean black hole solution in terms of the proper radial distance $\rho$ (in units of $l$ ), given by

$$
r=r_{+} \cosh (\rho)
$$

with $0 \leq \rho<\infty$, so that the Euclidean metric reads

$$
d s^{2}=\frac{r_{+}^{2}}{l^{2}} \sinh ^{2}(\rho) d \tau^{2}+l^{2} d \rho^{2}+r_{+}^{2} \cosh ^{2}(\rho) d \Sigma_{3}^{2}
$$

The thermodynamics of these kind of black holes turns out to be very sensitive to the geometry of the base manifold, this is briefly discussed in Section IV.

\section{B. Case (ii.b): Wormholes and spacetime horns}

In this case the base manifold possesses a constant Ricci scalar $\tilde{R}=6 \gamma$, with $\gamma$ normalized to \pm 1 or 0 .

Let us first consider the case for which the base manifold $\Sigma_{3}$ has nonvanishing Ricci scalar, i.e., $\gamma \neq 0$. By virtue of Eqs. (9), and (10) the spacetime metric (44) reads

$$
d s^{2}=-\left(\frac{r}{l}+a \sqrt{\frac{r^{2}}{l^{2}}+\gamma}\right)^{2} d t^{2}+\frac{d r^{2}}{\frac{r^{2}}{l^{2}}+\gamma}+r^{2} d \Sigma_{3}^{2},
$$

where $a$ is an integration constant and $l>0$. The Ricci scalar of (36) is given by

$$
R=-\frac{20}{l^{2}}-\frac{6 \gamma}{l}\left[r\left(\frac{r}{l}+a \sqrt{\frac{r^{2}}{l^{2}}+\gamma}\right)\right]^{-1},
$$

which generically diverges at $r=0$ and at any point satisfying $r / a<0$ and

$$
r_{s}^{2}=l^{2} \frac{\gamma a^{2}}{1-a^{2}}
$$

In the case $\gamma=1$ the metric possesses a timelike naked singularity at $r=0$, and if $-1<a<0$, an additional timelike naked singularity at $r^{2}=r_{s}^{2}$. Due to this ill geometrical behavior, we no longer consider the spacetime (36) for the case $\gamma=1$. 
- Wormholes: The case $\gamma=-1$ is much more interesting. The region $r<l$ must be excised since the metric (36) becomes complex within this range, and the Schwarzschild-like coordinates in (36) fail at $r=l$. Introducing the proper radial distance $\rho$, given by

$$
r=l \cosh (\rho)
$$

allows to extend the manifold beyond $r=l(\rho>0)$ to a geodesically complete manifold by letting $-\infty<\rho<\infty$. For $a^{2}<1$ the resulting metric for this geodesically complete manifold reads

$$
d s^{2}=l^{2}\left[-\cosh ^{2}\left(\rho-\rho_{0}\right) d t^{2}+d \rho^{2}+\cosh ^{2}(\rho) d \Sigma_{3}^{2}\right]
$$

where $\rho_{0}:=-\tanh ^{-1}(a)$, and the time coordinate has been rescaled. Note that since (36) is invariant under $(r, a) \rightarrow(-r,-a)$, the $\rho>0$ piece of (39) is isometric to (36) whereas the $\rho<0$ portion is isometric to the metric obtained by replacing $a \rightarrow-a$ in (36). In other words, (39) matches the region $r \geq l$ of the metric (36) with a given value of $a$, with the region $r \geq l$ of the same metric but reversing the sign of $a$. The singularity at $r^{2}=r_{s}^{2}$ in Eq. (38) is not present since $a^{2} \leq 1$, and that at $r=0$ is also absent since $r \geq l>0$ at all points.

For $a^{2}=1$ we obtain another wormhole in vacuum, by using again the proper distance $\rho$ defined above:

$$
d s^{2}=l^{2}\left[-e^{2 \rho} d t^{2}+d \rho^{2}+\cosh ^{2}(\rho) d \Sigma_{3}^{2}\right] .
$$

In these coordinates it is manifest that the metrics (39) and (40) describe wormholes, both possessing a throat located at $\rho=0$. No energy conditions are violated by these solutions, since in both cases, the whole spacetime is devoid of any kind of stress-energy tensor.

The spacetime described by Eq. (39) is the static wormhole solution found in [15]. This metric connects two asymptotically locally AdS regions, and gravity pulls towards a fixed hypersurface located at $\rho=\rho_{0}$ being parallel to the neck. This is revisited in the next subsection.

The metric (40) describes a brand new wormhole. Its Riemann tensor is given by 


$$
\begin{aligned}
& R_{t \rho}^{t \rho}=-\frac{1}{l^{2}}, R_{\rho j}^{\rho i}=-\frac{1}{l^{2}} \delta_{j}^{i}, R_{t j}^{t i}=-\frac{1}{l^{2}} \tanh (\rho) \delta_{j}^{i}, \\
& R_{k l}^{i j}=\frac{1}{l^{2}} \frac{\tilde{R}_{k l}^{i j} \cosh ^{2}(\rho)}{\cos ^{2}}-\frac{1}{l^{2}} \tanh ^{2}(\rho)\left(\delta_{k}^{i} \delta_{l}^{j}-\delta_{l}^{i} \delta_{k}^{j}\right),
\end{aligned}
$$

where latin indices run along the base manifold. At the asymptotic regions $\rho \rightarrow \pm \infty$ the curvature components approach

$$
\begin{aligned}
& R_{t \rho}^{t \rho}=-\frac{1}{l^{2}}, R_{\rho j}^{\rho i}=-\frac{1}{l^{2}} \delta_{j}^{i}, R_{t j}^{t i} \simeq \mp \frac{1}{l^{2}} \delta_{j}^{i}, \\
& R_{k l}^{i j} \simeq-\frac{1}{l^{2}}\left(\delta_{k}^{i} \delta_{l}^{j}-\delta_{l}^{i} \delta_{k}^{j}\right),
\end{aligned}
$$

This makes clear that the wormhole (40) connects an asymptotically locally AdS spacetime (at $\rho \rightarrow \infty)$ with another nontrivial smooth spacetime at the other asymptotic region $(\rho \rightarrow-\infty)$. Note that although the metric looks singular at $\rho \rightarrow-\infty$, the geometry is well behaved at this asymptotic region. This is seen by noting that the basic scalar invariants can be written in terms of contractions of the Riemann tensor with the index position as in (41), whose components have well defined limits (given in (42)), and $g^{\alpha}{ }_{\beta}=\delta^{\alpha}{ }_{\beta}$. Thus, the invariants cannot diverge. As an example, the limits of some invariants are

$$
\lim _{\rho \rightarrow-\infty} R_{\alpha \beta}^{\alpha \beta}=-\frac{8}{l^{2}}, \lim _{\rho \rightarrow-\infty} R_{\gamma \delta}^{\alpha \beta} R_{\alpha \beta}^{\gamma \delta}=\frac{40}{l^{4}}, \lim _{\rho \rightarrow-\infty} C^{\alpha \beta}{ }_{\gamma \delta} C^{\gamma \delta}{ }_{\alpha \beta}=\frac{8}{l^{4}}
$$

where $C^{\alpha \beta}{ }_{\gamma \delta}$ is the Weyl tensor.

We have also computed some differential invariants and found they are all well behaved as $\rho \rightarrow-\infty$.

Some features about the geodesics in these vacuum wormholes are discussed in the next subsection, their regularized Euclidean actions and their masses are evaluated in Sections $\mathrm{V}$ and $[\mathrm{IV}$, respectively.

- Spacetime horns: Let us consider now the case when the base manifold $\Sigma_{3}$ has vanishing Ricci scalar, i.e., $\tilde{R}=0$.

In this case the metric (41) reduces to

$$
d s^{2}=-\left(a \frac{r}{l}+\frac{l}{r}\right)^{2} d t^{2}+l^{2} \frac{d r^{2}}{r^{2}}+r^{2} d \Sigma_{3}^{2},
$$

where $a$ is an integration constant. The Ricci scalar of this spacetime reads

$$
R=-\frac{4}{l^{2}}\left(\frac{5 a r^{2}+l^{2}}{l^{2}+a r^{2}}\right) \text {. }
$$


The timelike naked singularity at $r_{s}^{2}=-\frac{l^{2}}{a}$ can be removed requiring $a \geq 0$; however this condition is not strong enough to ensure that the spacetime is free of singularities. Indeed the Kretschmann scalar is given by

$$
K:=R_{\lambda \rho}{ }^{\mu \nu} R_{\mu \nu}{ }^{\lambda \rho}=\frac{\tilde{R}_{k l}{ }^{i j} \tilde{R}_{i j}{ }^{k l}}{r^{4}}+\frac{8\left(5 r^{4} a^{2}+4 l^{2} r^{2} a+5 l^{4}\right)}{l^{4}\left(a r^{2}+l^{2}\right)^{2}},
$$

where $\tilde{R}_{k l}{ }_{l j} \tilde{R}_{i j}{ }^{k l}$ is the Kretchmann scalar of the Euclidean base manifold $\Sigma_{3}$. Hence, for a generic base manifold with vanishing Ricci scalar, the metric possesses a timelike naked singularity at $r=0$, unless the Kretchmann scalar of the base manifold vanishes. Since the base manifold is Euclidean, the vanishing of its Kretchmann scalar implies that it is locally flat. This drives us out of (ii.b1) to the degenerate case (ii.b2), for which the $g_{t t}$ component of the metric is not fixed by the field equations, for this reason we will not consider the locally flat case.

If the base manifold is not locally flat, at the origin the Ricci scalar goes to a constant and the Kretschmann scalar diverges as $r^{-4}$. Therefore, the singularity at the origin is smoother than that of a conifold [16], whose Ricci scalar diverges as $r^{-2}$, and it is also smoother than that of the five-dimensional Schwarzschild metric with negative mass, that possesses a timelike naked singularity at the origin with a Kretschmann scalar diverging as $r^{-8}$. In spite of this divergency, the regularized Euclidean action and the mass are finite for this solution, as we show in Sections IV and V. In this sense this singularity is as tractable as that of a vortex.

In the case $a>0$ we are interested in, we introduce $a=: e^{-2 \rho_{0}}$ and a time rescaling; then the metric (44) expressed in terms of the proper radial distance $r=l e^{\rho}$ is

$$
d s^{2}=l^{2}\left[-\cosh ^{2}\left(\rho-\rho_{0}\right) d t^{2}+d \rho^{2}+e^{2 \rho} d \Sigma_{3}^{2}\right] .
$$

This spacetime possesses a single asymptotic region at $\rho \rightarrow+\infty$ where it approaches AdS spacetime, but with a base manifold different from $S^{3}$. Note that as the warp factor of the base manifold goes to zero exponentially as $\rho \rightarrow-\infty$, it actually looks like a "spacetime horn".

For $a=0$, the metric (44) can also be brought into the form of a spacetime horn,

$$
d s^{2}=l^{2}\left[-e^{-2 \rho} d t^{2}+d \rho^{2}+e^{2 \rho} d \Sigma_{3}^{2}\right]
$$


which also possesses a single asymptotic region at $\rho \rightarrow+\infty$, which agrees with the asymptotic form of the new wormhole (40) as $\rho \rightarrow-\infty$.

The asymptotic form of the Riemann tensor is not that of a constant curvature manifold, and can then be obtained from the $\rho \rightarrow-\infty$ limit in (42).

The regularized Euclidean action and Mass of these spacetime horns are evaluated in Sections IV] and V]. Geodesics are discussed in the next subsection.

\section{Geodesics around wormholes and spacetime horns}

The class of metrics that describe the wormholes and spacetime horns is of the form

$$
d s^{2}=-A^{2}(\rho) d t^{2}+l^{2} d \rho^{2}+C^{2}(\rho) d \Sigma^{2}
$$

where the functions $A(\rho)$ and $C(\rho)$ can be obtained from Eqs. (39) and (40) for wormholes, and from Eqs.(47) and (48) for spacetime horns.

\section{Radial geodesics}

Let us begin with a brief analysis of radial geodesics for the wormholes and spacetime horns. The radial geodesics are described by the following equations

$$
\begin{aligned}
\dot{t}-\frac{E}{A^{2}} & =0, \\
l^{2} \dot{\rho}^{2}-\frac{E^{2}}{A^{2}}+b & =0,
\end{aligned}
$$

where dot stands for derivatives with respect to the proper time, the velocity is normalized as $u_{\mu} u^{\mu}=-b$, and the integration constant $E$ corresponds to the energy. As one expects, Eq. (51) tells that gravity is pulling towards the fixed hypersurface defined by $\rho=\rho_{0}$, where $\rho_{0}$ is a minimum of $A^{2}(\rho)$.

○ Wormholes: From (39) we have $A^{2}(\rho)=l^{2} \cosh ^{2}\left(\rho-\rho_{0}\right)$, then the equations for radial geodesics (50) and (51) reduce to

$$
\begin{aligned}
\dot{\rho}^{2}-\frac{E^{2}}{l^{4} \cosh ^{2}\left(\rho-\rho_{0}\right)} & =-\frac{b}{l^{2}}, \\
\dot{t}-\frac{E}{l^{2} \cosh ^{2}\left(\rho-\rho_{0}\right)} & =0 .
\end{aligned}
$$


These equation immediately tell us that [15]: The $\rho$ coordinate of a radial geodesic behaves as a classical particle in a Pöschl-Teller potential; timelike geodesics are confined, they oscillate around the hypersurface $\rho=\rho_{0}$. An observer sitting at $\rho=\rho_{0}$ lives in a timelike geodesic (here $d \tau / d t=l, \tau$ the proper time of this static observer); radial null geodesics connect both asymptotic regions (i.e., $\rho=-\infty$ with $\rho=+\infty$ ) in a finite $t$ span $\Delta t=\pi$, which does not depend on $\rho_{0}$ (the static observer at $\rho=\rho_{0}$ says that this occurred in a proper time $\Delta \tau=\pi l$ ). These observations give a meaning to $\rho_{0}$ : gravity is pulling towards the fixed hypersurface defined by $\rho=\rho_{0}$, which is parallel to the neck at $\rho=0$, and therefore $\rho_{0}$ is a modulus parameterizing the proper distance from this hypersurface to the neck.

The geodesic structure of the new wormhole (40) is quite different from the previous one. In this case, the radial geodesic Eqs. (50) and (51) read

$$
\begin{aligned}
& \dot{\rho}^{2}-\frac{e^{-2 \rho} E^{2}}{l^{4}}=-\frac{b}{l^{2}}, \\
& l^{2} \dot{t}-e^{-2 \rho} E=0 .
\end{aligned}
$$

As expected, the behavior of the geodesics at $\rho \rightarrow+\infty$ is like in an AdS spacetime. Moreover, since gravity pulls towards the asymptotic region $\rho \rightarrow-\infty$, radial timelike geodesics always have a turning point and they are doomed to approach to $\rho \rightarrow-\infty$ in the future. Note that the proper time that a timelike geodesic takes to reach the asymptotic region at $\rho=-\infty$, starting from $\rho=\rho_{f}$ is finite and given by

$$
\Delta \tau=\int_{\rho=-\infty}^{\rho=\rho_{f}} \frac{l^{2} d \rho}{\sqrt{E^{2} e^{-2 \rho}-l^{2}}}=\frac{\pi l}{2}-l \tan ^{-1}\left(\sqrt{\frac{E^{2}}{l^{2}} e^{-2 \rho_{f}}-1}\right)<\infty .
$$

It is easy to check that null radial geodesics can also reach the asymptotic region at $\rho=$ $-\infty$ in a finite affine parameter. This, together with the fact that spacetime is regular at this boundary, seems to suggest that it could be analytically continued through this surface. However, since the warp factor of the base manifold blows up at $\rho=-\infty$, this null hypersurface should be regarded as a spacetime boundary.

- Spacetime horns: For the spacetime horn (47), the $(\rho, t)$ piece of the metric agrees with that of the wormhole (39). Hence, the structure of radial geodesics in both cases is the same, with gravity pulling towards the $\rho=\rho_{0}$ surface. Timelike geodesics again have a turning point, which, in this case, prevents the geodesics from hitting the singularity at $\rho=-\infty$. 
In the case of the spacetime horn (48) (compare to (40)), gravity becomes a repulsive force pointing from the singularity at $\rho \rightarrow-\infty$, towards the asymptotic region at $\rho \rightarrow+\infty$. Therefore timelike radial geodesics are doomed to end up at the asymptotic region in a finite proper time (see (56) )

\section{Gravitational vs. centrifugal forces}

In this Section we discuss an interesting effect that occurs for geodesics with nonzero angular momentum. One can see that for the generic class of spacetimes (49), which includes wormholes and spacetime horns, there is a region where the gravitational and centrifugal effective forces point in the same direction. These are expulsive regions that have a single turning point for any value of the conserved energy, and within which bounded geodesics cannot exist.

The class of metrics we consider are (49) with the further restriction that the base manifold $\Sigma_{3}$ have a Killing vector $\xi$. Choosing adapted coordinates $y=\left(x^{1}, x^{2}, \phi\right)$ such that $\xi=\partial / \partial \phi$, the base manifold metric is $d \Sigma_{3}^{2}=\tilde{g}_{i j}(x) d y^{i} d y^{j}$ and the spacetime geodesics with $x$ fixed are described by the following equations

$$
\begin{aligned}
\dot{t} & =\frac{E}{A^{2}} ; \dot{\phi}=\frac{L}{C^{2}} \\
l^{2} \dot{\rho}^{2} & =-b+\frac{E^{2}}{A^{2}}-\frac{L^{2}}{C^{2}} .
\end{aligned}
$$

Here we have used the fact that, if $u^{a}$ is the geodesic tangent vector, then $\xi^{a} u_{a}=\mathcal{L}$ is conserved, and $\dot{\phi}=\mathcal{L} /\left(C^{2} \tilde{g}_{\phi \phi}(x)\right)=: L / C^{2}$. If $\xi$ is a $U(1)$ Killing vector then $\mathcal{L}$ is a conserved angular momentum. Examples are not hard to construct, for spacetime horns, what we need is a base manifold with zero Ricci scalar and a $U(1)$ Killing field. For wormholes, we need a nonflat 3-manifold with $\tilde{R}=-6$ and a $U(1)$ isometry, an example being $S^{1} \times H_{2} / \Gamma$, where $\Gamma$ is a freely acting discrete subgroup of $O(2,1)$, and the metric locally given by:

$$
d \Sigma_{3}^{2}=\frac{1}{3}\left(d x_{1}^{2}+\sinh ^{2}\left(x_{1}\right) d x_{2}^{2}\right)+d \phi^{2} .
$$

The motion along the radial coordinate in proper time is like that of a classical particle in an effective potential given by the r.h.s. of Eq. (57). This effective potential, has a minimum at $\rho=\bar{\rho}$ only if the following condition is fulfilled

$$
\frac{A^{\prime}(\bar{\rho})}{A(\bar{\rho})^{3}} E^{2}=\frac{C^{\prime}(\bar{\rho})}{C(\bar{\rho})^{3}} L^{2} .
$$


This expresses the fact that the gravitational effective force is canceled by the centrifugal force if the orbit sits at $\rho=\bar{\rho}$. The class of spacetimes under consideration have regions $\mathcal{U}$ where the sign of $A^{-3} A^{\prime}$ is opposite to that of $C^{-3} C^{\prime}$, i.e., the effective gravitational and centrifugal forces point in the same direction. Within these regions, there is at most a single turning point, and consequently bounded orbits cannot exist.

In the case of a wormhole (39), Eq. (59) reads

$$
\frac{E^{2} \tanh \left(\bar{\rho}-\rho_{0}\right)}{\cosh ^{2}\left(\bar{\rho}-\rho_{0}\right)}=\frac{L^{2} \tanh \bar{\rho}}{\cosh ^{2} \bar{\rho}} .
$$

The centrifugal force reverses its sign at the neck at $\rho=0$, the Newtonian force does it at $\rho=\rho_{0}$, both forces being aligned for $\rho$ between zero and $\rho_{0}$. The expulsive region $\mathcal{U}$ is nontrivial whenever $\rho_{0} \neq 0$. This situation is depicted in Fig. 1a.

In the case of the new wormhole solution (40) the region $\mathcal{U}$ is defined $\rho \leq 0$ (See Fig. 1b), and for the spacetime horn (47) the region $\mathcal{U}$ is given by $\rho \leq \rho_{0}$ (Fig. 1c). Finally, for the spacetime horn (48) the region $\mathcal{U}$ is the entire spacetime, there are no bounded geodesics.

\section{REGULARIZED EUCLIDEAN ACTION}

Here it is shown that the geometrically well-behaved solutions discussed in the previous Section have finite Euclidean action, which reduces to the free energy in the case of black holes, and vanishes for the other solutions.

The action (2) in the case of special choice of coefficients can be written as

$$
I_{5}=\kappa \int_{M} \epsilon_{a b c d e}\left(R^{a b} R^{c d}+\frac{2}{3 l^{2}} R^{a b} e^{c} e^{d}+\frac{1}{5 l^{4}} e^{a} e^{b} e^{c} e^{d}\right) e^{e},
$$

and it has been shown that it can be regularized by adding a suitable boundary term in a background independent way, which depends only on the extrinsic curvature and the geometry at the boundary [17]. The total action then reads

$$
I_{T}=I_{5}-B_{4}
$$

where the boundary term is given by

$$
B_{4}=\kappa \int_{\partial M} \epsilon_{a b c d e} \theta^{a b} e^{c}\left(R^{d e}-\frac{1}{2} \theta^{d}{ }_{f} \theta^{f e}+\frac{1}{6 l^{2}} e^{d} e^{e}\right),
$$




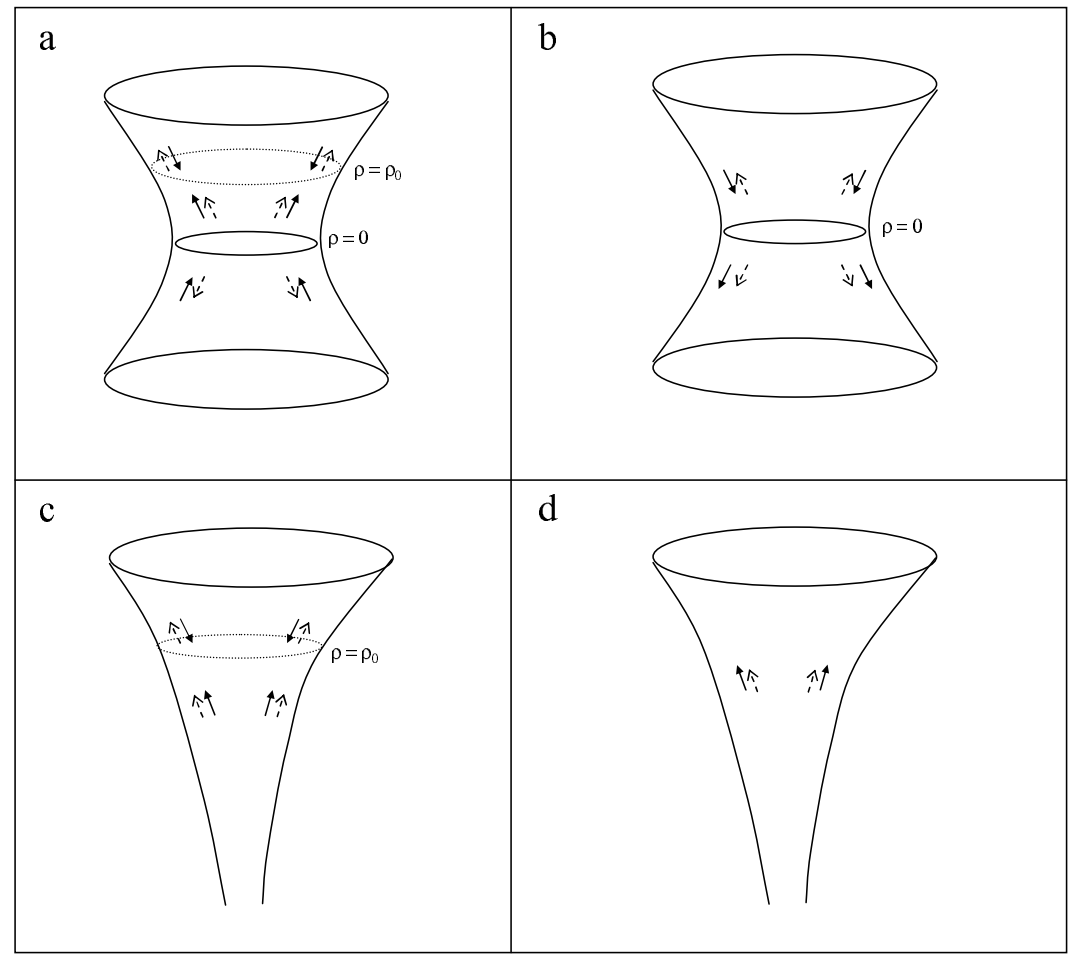

FIG. 1: Gravitational vs. centrifugal forces for wormholes and spacetime horns. In this diagram, black and dashed arrows represent effective gravitational and centrifugal forces, respectively. Figures a and b, correspond to the wormholes (39) and (40), while figures c and d represent the spacetime horns (47) and (48), respectively.

and $\theta^{a b}$ is the second fundamental form. The total action (62) attains an extremum for solutions of the field equations provided

$$
\delta I_{T}=\kappa \int_{\partial M} \epsilon_{a b c d e}\left(\delta \theta^{a b} e^{c}-\theta^{a b} \delta e^{c}\right)\left(\bar{R}^{d e}-\frac{1}{2} \theta_{f}^{d} \theta^{f e}-\frac{1}{2 l^{2}} e^{d} e^{e}\right)=0,
$$

where $\bar{R}^{a b}:=R^{a b}+\frac{1}{l^{2}} e^{a} e^{b}$. Therefore, the value of the regularized Euclidean action makes sense for solutions which are bona fide extrema, i.e., for solutions such that condition (64) is fulfilled.

The Euclidean continuation of the class of spacetimes described in Section III, including black holes, wormholes and spacetime horns, is described by metrics of the form

$$
d s^{2}=A^{2}(\rho) d \tau^{2}+l^{2} d \rho^{2}+C^{2}(\rho) d \Sigma_{3}^{2},
$$

where $0 \leq \tau \leq \beta$ is the Euclidean time, and the functions $A$ and $C$ correspond to the ones appearing in Eq. (35) for the black holes; Eqs. (39) and (40) for the wormholes, and in Eqs. (47) and (48) for the spacetime horns. 
Let us first check that these solutions are truly extrema of the total action (62).

\section{A. Geometrically well-behaved solutions as extrema of the regularized action}

For the class of solutions under consideration, the curvature two-form satisfies

$$
\bar{R}^{01}=\bar{R}^{1 m}=0
$$

and the condition (64) reduces to

$$
\delta I_{T}=\kappa \beta\left[F \mathcal{I}_{3}+6 G \mathcal{V}_{3}\right]_{\partial \Sigma}
$$

where $\beta$ is the Euclidean time period, $\mathcal{V}_{3}$ is the volume of the base manifold, and $\partial \Sigma$ is the boundary of the spatial section. In Eq. (67) $\mathcal{I}_{3}$ is defined by

$$
\mathcal{I}_{3}:=\int_{\Sigma_{3}} \sqrt{\tilde{g}} \tilde{R} d^{3} x
$$

and the functions $F$ and $G$ in (67) are given by

$$
\begin{aligned}
F & :=\frac{2}{l}\left[A^{\prime} \delta C-A \delta C^{\prime}+C^{\prime} \delta A-C \delta A^{\prime}\right] \\
G & :=\left[A^{\prime}\left(C^{2}-C^{2}\right)+2 C^{\prime}\left(C A-C^{\prime} A^{\prime}\right)\right] \frac{\delta C}{l^{3}} \\
& -\left[A\left(C^{2}-C^{\prime 2}\right)+2 C\left(C A-C^{\prime} A^{\prime}\right)\right] \frac{\delta C^{\prime}}{l^{3}} \\
& +C^{\prime}\left(C^{2}-C^{\prime 2}\right) \frac{\delta A}{l^{3}}-C\left(C^{2}-C^{2}\right) \frac{\delta A^{\prime}}{l^{3}}
\end{aligned}
$$

Here we work in the minisuperspace approach, where the variation of the functions $A$ and $C$ correspond to the variation of the integration constants, and prime $\left(^{\prime}\right)$ denotes derivative with respect to $\rho$.

Now it is simple to evaluate the variation of the action (67) explicitly for each case.

- Black holes: As explained in Section III, the Euclidean black hole metric is given by

$$
d s^{2}=\frac{r_{+}^{2}}{l^{2}} \sinh ^{2}(\rho) d \tau^{2}+l^{2} d \rho^{2}+r_{+}^{2} \cosh ^{2}(\rho) d \Sigma_{3}^{2}
$$

with $\beta=\frac{2 \pi l^{2}}{r_{+}}$, and it has a single boundary which is of the form $\partial M=S^{1} \times \Sigma_{3}$. In order to evaluate (67) it is useful to introduce the regulator $\rho_{a}$, such that $0 \leq \rho \leq \rho_{a}$. It is easy to verify that the functions $F$ and $G$ defined in (69) and (70) respectively, satisfy

$$
F\left(\rho_{a}\right)=G\left(\rho_{a}\right)=0
$$


and hence, the boundary term (67) identically vanishes. Note that it was not necessary to take the limit $\rho_{a} \rightarrow+\infty$.

- Wormholes: The Euclidean continuation of both wormhole solutions in Eqs. (39) and (40) can be written as

$$
d s^{2}=l^{2}\left[(\cosh \rho+a \sinh \rho)^{2} d \tau^{2}+d \rho^{2}+\cosh ^{2} \rho d \Sigma_{3}^{2}\right]
$$

where the metrics (39) and (40) are recovered for $a^{2}<1$ and $a^{2}=1$, respectively, and $\beta$ is arbitrary. In this sense, the wormhole (40) can be regarded as a sort of extremal case of the wormhole (39). In this case, since the boundary is of the form $\partial \Sigma=\Sigma_{3}^{+} \cup \Sigma_{3}^{-}$it is useful to introduce the regulators $\rho_{ \pm}$, such that $\rho_{-} \leq \rho \leq \rho_{+}$. Using the fact that the base manifold has a negative constant Ricci scalar given by $\tilde{R}=-6$, the variation of the action (67) reduces to

$$
\delta I_{T}=6 \kappa \beta l \delta a\left[\mathcal{V}_{3}\right]_{\rho_{-}}^{\rho_{+}}=0
$$

Note that, as in the case for the black hole, the boundary term vanishes regardless the position of the regulators $\rho_{-}$and $\rho_{+}$.

- Spacetime horns: The Euclidean continuation of the spacetime horns in Eqs. (47) and (48) can be written as

$$
d s^{2}=l^{2}\left[\left(a e^{\rho}+e^{-\rho}\right)^{2} d \tau^{2}+d \rho^{2}+e^{2 \rho} d \Sigma_{3}^{2}\right],
$$

with an arbitrary time period $\beta$. The metrics (47) and (48) are recovered for $a>0$ and $a=0$, respectively. From this one see that (48) is a kind of extremal case of (47). In this case, as $\rho \rightarrow+\infty$, the spacetime has a boundary of the form $\partial M=S^{1} \times \Sigma_{3}$. Since generically, there is a smooth singularity when $\rho \rightarrow-\infty$, it is safer to introduce two regulators $\rho_{ \pm}$, satisfying $\rho_{-} \leq \rho \leq \rho_{+}$. Due to the fact that the base manifold has vanishing Ricci scalar, only the second term at the r.h.s. of Eq. (67) remains, i.e.,

$$
\delta I_{T}=6 \kappa \beta\left[G \mathcal{V}_{3}\right]_{\rho_{-}}^{\rho_{+}}
$$

and it is simple to check that, since $G\left(\rho_{-}\right)=G\left(\rho_{+}\right)=0$ the boundary term (67) vanishes again regardless the position of the regulators.

In sum, as we have shown that the black holes, wormholes and spacetime horns are truly extrema of the action, it makes sense to evaluate the regularized action on these solutions. 


\section{B. Euclidean action for geometrically well-behaved solutions}

For the class of solutions of the form (65), which satisfy (66), the bulk and boundary contributions to the regularized action $I_{T}=I_{5}-B_{4}$, given by Eqs. (61) and (63) respectively, reduce to

$$
\begin{aligned}
& I_{5}=\kappa \beta\left[\begin{array}{llll}
H & \mathcal{I}_{3}+6 & J & \mathcal{V}_{3}
\end{array}\right], \\
& B_{4}=\kappa \beta\left[\begin{array}{llll}
h & \mathcal{I}_{3}+6 & j & \mathcal{V}_{3}
\end{array}\right]_{\partial \Sigma} .
\end{aligned}
$$

The functions $H$ and $J$ in the bulk term are defined by

$$
\begin{aligned}
H & :=-\frac{8}{l} \int A C d \rho, \\
J & :=\frac{4}{l^{3}} \int\left[\left(C^{2}\right)^{\prime}(A C)^{\prime}-\frac{4}{3} A C^{3}\right] d \rho,
\end{aligned}
$$

where the integrals are taken along the whole range of $\rho$. For the boundary term (777), the functions $h$ and $j$ are respectively defined by

$$
\begin{aligned}
& h=-\frac{2}{l}(A C)^{\prime}, \\
& j=-\frac{1}{l^{3}}\left[(A C)^{\prime}\left(\frac{C^{2}}{3}-C^{2}\right)+\left(C^{2}\right)^{\prime}\left(\frac{A C}{3}-A^{\prime} C^{\prime}\right)\right] .
\end{aligned}
$$

Now it is straightforward to evaluate the regularized Euclidean action for the class of solutions under consideration.

- Black holes: In order to obtain the regularized Euclidean action for the black hole (35) one introduces the regulator $\rho_{a}$, such that the range of the proper radial distance is given by $0 \leq \rho \leq \rho_{a}$. The regularized action $I_{T}$ for the black hole is

$$
I_{T}=4 \pi \kappa r_{+}\left[\mathcal{I}_{3}+\frac{r_{+}^{2}}{l^{2}} \mathcal{V}_{3}\right]
$$

Note that the action is finite and independent of the regulator $\rho_{a}$.

For a fixed temperature, the Euclidean action (82) is related to the free energy $F$ in the canonical ensemble as

$$
I_{T}=-\beta F=S-\beta M
$$

so that the mass and the entropy can be obtained from

$$
M=-\frac{\partial I_{T}}{\partial \beta} ; S=\left(1-\beta \frac{\partial}{\partial \beta}\right) I_{T} .
$$


In the case of a generic base manifold $\Sigma_{3}$, the thermodynamics of the black holes in Eq. (35) turns out to be qualitatively the same as the one described in Ref. [13]. In the case of base manifolds of constant curvature it agrees with previously known results.

Note that the mass of the black hole:

$$
M=2 \kappa \frac{r_{+}^{2}}{l^{2}}\left[\mathcal{I}_{3}+\frac{3 r_{+}^{2}}{l^{2}} \mathcal{V}_{3}\right]
$$

is very sensitive to the geometry of the base manifold. For a fixed base manifold with $\mathcal{I}_{3}<0$, $M$ is bounded from below by $M_{0}:=-\frac{\kappa}{6} \frac{\mathcal{I}_{3}^{2}}{\mathcal{V}_{3}}$. Note that $M_{0}$ can be further minimized due to the freedom in the choice of the base manifold. Even more interesting is the fact that, among the solutions with a given base manifold satisfying $\mathcal{I}_{3}<0$, the Euclidean action (82) has a minimum value, attained at

$$
r_{+}=l \sqrt{\frac{-\mathcal{I}_{3}}{3 \mathcal{V}_{3}}},
$$

that can be written in terms of the Yamabe functional $Y_{3}:=\frac{\mathcal{I}_{3}}{\mathcal{V}_{3}^{1 / 3}}[18]$

$$
I_{T_{0}}=-\frac{8 \sqrt{3}}{9} \pi \kappa l\left|Y_{3}\right|^{3 / 2}
$$

Note that the freedom in the choice of the boundary metric allows further minimization of the extremum of the action (87). This can be performed by choosing $\Sigma_{3}$ as a stationary point of the Yamabe functional. Since it is well known that the Yamabe functional has critical points for Einstein metrics, and three-dimensional Einstein metrics are metrics of constant curvature, the base manifold turns out to be of negative constant curvature.

- Wormholes: The Euclidean continuation of the wormhole metrics (39) and (40) are smooth independently of the Euclidean time period $\beta$. The Euclidean action $I_{T}=I_{5}-B_{4}$, is evaluated introducing regulators such that $\rho_{-} \leq \rho \leq \rho_{+}$.

In the case of the Euclidean wormhole (39) the regularized Euclidean action vanishes regardless the position of the regulators, since

$$
I_{5}=B_{4}=2 \kappa l \beta \mathcal{V}_{3}\left[3 \sinh \left(\rho_{0}\right)+8 \cosh ^{3}(\rho) \sinh \left(\rho-\rho_{0}\right)\right]_{\rho_{-}}^{\rho_{+}} .
$$

Consequently, the mass of this spacetime also vanishes, since $M=-\frac{\partial I_{T}}{\partial \beta}=0$.

For the wormhole (40) the Euclidean action reads

$$
I_{T}=6 \kappa \beta \mathcal{V}_{3}[(J-j)-(H-h)]
$$


with

$$
\begin{aligned}
H & =-\left.2 l\left(e^{2 \rho}+2 \rho\right)\right|_{\rho_{-}} ^{\rho_{+}}, \\
J & =-\left.\frac{1}{3} l\left(-e^{4 \rho}+3 e^{2 \rho}+12 \rho-e^{-2 \rho}\right)\right|_{\rho_{-}} ^{\rho_{+}}, \\
h & =-\left.2 l e^{2 \rho}\right|_{\rho_{-}} ^{\rho_{+}}, \\
j & =-\left.\frac{1}{3} l\left(-e^{-4 \rho}+3 e^{2 \rho}-e^{-2 \rho}\right)\right|_{\rho_{-}} ^{\rho_{+}} .
\end{aligned}
$$

The regularized action vanishes again independently of $\rho_{ \pm}$, and so does it mass.

It is worth pointing out that both wormholes can be regarded as instantons with vanishing Euclidean action.

- Spacetime horns: The Euclidean continuation of the spacetime horns (47) and (48) have arbitrary $\beta$. Let us recall that when $\rho \rightarrow+\infty$, the spacetime has a boundary of the form $\partial M=S^{1} \times \Sigma_{3}$, and due to the presence of the singularity at $\rho \rightarrow-\infty$, we introduce regulators $\rho_{ \pm}$, such that $\rho_{-} \leq \rho \leq \rho_{+}$. Since the Ricci scalar of $\Sigma_{3}$ vanishes, the regularized action for the spacetime horns reduce to

$$
I_{T}=6 \kappa \beta \mathcal{V}_{3}(J-j)
$$

For the spacetime horn (47), the Euclidean action

$$
\begin{aligned}
J & =\left.\frac{4}{3} l\left(e^{4 \rho+\rho_{0}}-e^{2 \rho-\rho_{0}}\right)\right|_{\rho_{-}} ^{\rho_{+}}, \\
j & =\left.\frac{4}{3} l\left(e^{4 \rho+\rho_{0}}-e^{2 \rho-\rho_{0}}\right)\right|^{\rho_{+}} .
\end{aligned}
$$

vanish. Note that it was necessary to take the limit $\rho_{-} \rightarrow-\infty$.

In the case of the spacetime horn (48), in the limit $\rho_{-} \rightarrow-\infty$, the regularized action also vanishes since

$$
\begin{gathered}
J=-\left.\frac{8}{3} l e^{2 \rho}\right|_{\rho_{-}} ^{\rho_{+}}, \\
j=-\left.\frac{8}{3} l e^{2 \rho}\right|^{\rho_{+}} .
\end{gathered}
$$

As a consequence, the masses of the spacetime horns vanishes.

The mass for the spacetime metrics discussed here can also be obtained from a suitable surface integral coming from a direct application of Noether's theorem to the regularized action functional. 


\section{MASS FROM A SURFACE INTEGRAL}

As in section IV it was shown that the geometrically well behaved solutions are truly extrema of the regularized action, one is able to compute the mass from the following surface integral

$$
Q(\xi)=\frac{\kappa}{l} \int_{\partial \Sigma} \epsilon_{a b c d e}\left(I_{\xi} \theta^{a b} e^{c}+\theta^{a b} I_{\xi} e^{c}\right)\left(\tilde{R}^{d e}+\frac{1}{2} \theta_{f}^{d} \theta^{f e}+\frac{1}{2 l^{2}} e^{d} e^{e}\right),
$$

obtained by the straightforward application of Noether's theorem ${ }^{3}$. Here $\xi=\partial_{t}$ is the timelike Killing vector.

For a metric of the form (65), satisfying (66), (196) gives

$$
M=2 \frac{\kappa}{l}\left[\left(A^{\prime} C-C^{\prime} A\right)\left(\mathcal{I}_{3}+\frac{3}{l^{2}}\left(C^{2}-C^{\prime 2}\right) \mathcal{V}_{3}\right)\right]_{\partial \Sigma},
$$

which can be explicitly evaluated for the black holes, wormholes and spacetime horns.

○ Black holes: For the black hole metric (33) the mass in Eq. (97) reads

$$
M=2 \kappa \frac{r_{+}^{2}}{l^{2}}\left[\mathcal{I}_{3}+\frac{3 r_{+}^{2}}{l^{2}} \mathcal{V}_{3}\right]
$$

It is reassuring to verify that it coincides with the mass computed within the Euclidean approach in Eq. (85).

- Wormholes: As explained in Ref.[15], for the wormhole (39), one obtains that the contribution to the total mass coming from each boundary reads

$$
M_{ \pm}=Q_{ \pm}\left(\partial_{t}\right)= \pm 6 \kappa \mathcal{V}_{3} \sinh \left(\rho_{0}\right)
$$

where $Q_{ \pm}\left(\partial_{t}\right)$ is the value of (96) at $\partial \Sigma_{ \pm}$, which again does not depend on $\rho_{+}$and $\rho_{-}$. The opposite signs of $M_{ \pm}$, are due to the fact that the boundaries of the spatial section have opposite orientation. The integration constant $\rho_{0}$ can be regarded as a parameter for the apparent mass at each side of the wormhole, which vanishes only when the solution acquires reflection symmetry, i.e., for $\rho_{0}=0$. This means that for a positive value of $\rho_{0}$, the mass of the wormhole appears to be positive for observers located at $\rho_{+}$, and negative for the ones at $\rho_{-}$, with a vanishing total mass $M=M_{+}+M_{-}=0$.

\footnotetext{
3 The action of the contraction operator $I_{\xi}$ over a $p$-form $\alpha_{p}=\frac{1}{p !} \alpha_{\mu_{1} \cdots \mu_{p}} d x^{\mu_{1}} \cdots d x^{\mu_{p}}$ is given by $I_{\xi} \alpha_{p}=$
} $\frac{1}{(p-1) !} \xi^{\nu} \alpha_{\nu \mu_{1} \cdots \mu_{p-1}} d x^{\mu_{1}} \cdots d x^{\mu_{p-1}}$, and $\partial \Sigma$ stands for the boundary of the spacelike section. 
For the wormhole (40) the total mass also vanishes since the contribution to the surface integral (96) coming from each boundary reads

$$
M_{ \pm}=\mp 6 \kappa \mathcal{V}_{3}
$$

so that $M=M_{+}+M_{-}=0$.

Note that $M_{ \pm}$are concrete examples of Wheeler's conception of "mass without mass".

- Spacetime horns: For the spacetime horns (47) and (48) the masses also vanish. This can be easily verified from (97), the fact that $\mathcal{I}_{3}=0$ (since $\tilde{R}=0$ ), and that the warp factor of the base manifold, $C=e^{\rho}$, satisfies $\left(C^{2}-C^{\prime 2}\right)=0$.

\section{DISCUSSION AND COMMENTS}

An exhaustive classification for the class of metrics (4) which are solutions of the EinsteinGauss-Bonnet theory in five dimensions has been performed. In Section II, it was shown that for generic values of the coupling constants, the base manifold $\Sigma_{3}$ must be necessarily of constant curvature, and consequently, the solution reduces to the topological extension of the Boulware-Deser metric, for which $f^{2}=g^{2}$ is given by (6) . It has also been shown that the base manifold admits a wider class of geometries for those special theories for which the Gauss-Bonnet coupling acquires a precise relation in terms of the cosmological and Newton constants, given by (7).

Remarkably, the additional freedom in the choice of the metric at the boundary, which determines $\Sigma_{3}$, allows the existence of three main branches of geometries in the bulk (Section II).

The geometrically well-behaved metrics among this class correspond to the case of negative cosmological constant.

If the boundary metric is chosen to be such that $\Sigma_{3}$ is an arbitrary, but fixed, base manifold, the solution is given by (33), and describes black holes whose horizon geometry inherits the metric of the base manifold. These solutions generalize those in [12] and [13], for which $\Sigma_{3}$ was assumed to be of constant curvature, which, in the case of spherical symmetry, reduce to the metrics in [9], [14].

If the metric at the boundary is chosen so that the base manifold $\Sigma_{3}$ possesses a constant negative Ricci scalar, two different kinds of wormhole solutions in vacuum are obtained. One 
of them, given in (39), was found previously in [15] and describes a wormhole connecting two asymptotic regions whose metrics approach that of AdS spacetime, but with a different base manifold. The other solution, given in (40), describes a brand new wormhole connecting an asymptotically locally AdS spacetime at one side of the throat, with a nontrivial curved and smooth spacetime on the other side. Note that, in view of Yamabe's theorem [18], any compact Riemannian manifold has a conformally related Riemannian metric with constant Ricci scalar, so that there are many possible choices for $\Sigma_{3}$.

For boundary metrics for which the base manifold $\Sigma_{3}$ has vanishing Ricci scalar, a different class of solutions is shown to exist. For these "spacetime horns" the warp factor of the base manifold is an exponential of the proper radial distance, and generically possess a singularity as $\rho \rightarrow-\infty$. As explained in Sec. III, this singularity is weaker than that of the five-dimensional Schwarzschild solution with negative mass, and it is also weaker than that of a conifold.

It has also been shown that if $\Sigma_{3}$ is of constant curvature, due to certain class of degeneration of the field equations for the theories satisfying (7), there is a special case where the metric admits an arbitrary redshift function. This degeneracy is a known feature of the class of theories considered here [19]. A similar degeneracy has been found in the context of Birkhoff's theorem for the Einstein-Gauss-Bonnet theory [20], [21], which cannot be removed by a coordinate transformation [22]. Birkhoff's theorem has also been discussed in the context of theories contaning a dilaton and an axion field coupled with a Gauss-Bonnet term in [23].

In the sense of the AdS/CFT correspondence [24], the dual CFT living at the boundary, which in our case is of the form $S^{1} \times \Sigma_{3}$, should acquire a radically different behavior according to the choice of $\Sigma_{3}$, since it has been shown that the bulk metric turns out to be very sensitive to the geometry of the base manifold. Notice that the existence of asymptotically AdS wormholes raises some puzzles concerning the AdS/CFT conjecture [25], [26], [27].

It is worth pointing out that an interesting effect occurs for geodesics with angular momentum for the generic class of spacetimes given by (49), among which the wormholes and spacetime horns are included. In a few words, there are regions for which the effective potential cannot have a minimum, since the gravitational force points in the same direction as the centrifugal force. Therefore, within these regions, there is at most a single turning 
point, and consequently bounded orbits cannot exist.

In Sec. IV, it was shown that the geometrically well-behaved solutions have finite Euclidean action. In the case of black holes, the Euclidean action reduces to the free energy in the canonical ensemble. It has also been shown that black holes whose base manifolds are such that its Einstein-Hilbert action $\mathcal{I}_{3}$ is negative, have a nontrivial ground state, for which its Euclidean action is an increasing function of the Yamabe functional, and therefore, its value is further extremized when the base manifold $\Sigma_{3}$ is of constant curvature.

In the case of wormholes, the Euclidean continuation is regular for an arbitrary Euclidean time period $\beta$, and they can be regarded as instantons with vanishing Euclidean action and mass. For the spacetime horns, their regularized action and mass vanish; so that in this sense, the singularity is as tractable as it is for a vortex.

It is simple to see that, the class of solutions discussed here can be embedded into the locally supersymmetric extension of the five-dimensional Einstein-Gauss-Bonnet for the choice of coefficients (77) [28], [29]. As a consequence, the black holes (33) admit a ground state with unbroken supersymmetries whose Killing spinors were explicitly obtained in [30]. In this case the base manifold must necessarily be Einstein.

For the special coefficients (17), the freedom in the choice of the base manifold allows to consider as a particular case, base manifolds of the form $\Sigma_{3}=S^{1} \times \Sigma_{2}$. This can be performed for all the branches, but not for the degenerate one. This means that compactification to four dimensions for the black holes (33), the wormholes (39), (40), and the spacetime horns (47), (48) is straightforward. Therefore, the dimensionally reduced solutions posses the same causal behavior as their five-dimensional seeds, but they are supported by a nontrivial dilaton field with a nonvanishing stress-energy tensor. Further compactifications have been found in Refs. [31], [32] and [33]. The dimensional reduction of the Einstein-Gauss-Bonnet theory has been discussed in Ref. [34], and for the special choice of coefficients (7), it has been discussed recently in Ref. [35], including new exact solutions.

For the Einstein-Gauss-Bonnet theory, black holes with nontrivial horizon geometry have also been discussed in Refs. [36], [37]; it is worth pointing out that the stability of GaussBonnet black holes is fairly different than that of the Schwarszchild solution [38], [39], [40], [41], [42]. Solutions possessing NUT charge have been found in [43]. Wormhole solutions for this theory, in the presence of matter that does not violate the weak energy condition 
have been shown to exist provided the Gauss-Bonnet coupling constant is negative and bounded according to the shape of the solution [44]. Thin shells wormholes for this theory have been discussed recently in [45]. For the pure Gauss-Bonnet theory, i.e., for the action (2) with $\alpha_{1}=\alpha_{0}=0$, wormhole solutions in vacuum, for which there is a jump in the extrinsic curvature along a spacelike surface, have been shown to exist recently [46]. Higher dimensional wormhole solutions have also been discussed in the context of braneworlds, see e.g., [47] and references therein.

As a final remark, it is worth pointing out that the results found here are not peculiarities of five-dimensional gravity, and similar structures can be found in higher dimensional spacetimes [48].

Acknowledgments.- We thank Arturo Gómez for thorough reading of this paper and for useful remarks. G.D is supported by CONICET. J.O. thank the support of projects MECESUP UCO-0209 and MECESUP USA-0108. J. O. and R. T. thanks the organizers of "Grav06, Fifty years of FaMAF \& Workshop on Global Problems in GR", held in Córdoba, for their warm hospitality. This work was partially funded by FONDECYT grants 1040921, 1051056, 1061291, 1071125; Secyt-UNC and CONICET. This work was funded by an institutional grant to CECS of the Millennium Science Initiative, Chile and also benefits from the generous support to CECS by Empresas CMPC.

[1] D. Lovelock, J. Math. Phys. 12, 498 (1971).

[2] D. Birmingham, Class. Quant. Grav. 16, 1197 (1999).

[3] G. Gibbons and S. A. Hartnoll, Phys. Rev. D 66, 064024 (2002).

[4] J. P. S. Lemos, Phys. Lett. B 353, 46 (1995);

[5] L. Vanzo, Phys. Rev. D 56, 6475 (1997)

[6] D. R. Brill, J. Louko and P. Peldan, Phys. Rev. D 56, 3600 (1997).

[7] G. Dotti and R. J. Gleiser, Phys. Lett. B 627, 174 (2005).

[8] R. G. Cai, Phys. Rev. D 65, 084014 (2002).

[9] D. G. Boulware and S. Deser, Phys. Rev. Lett. 55, 2656 (1985).

[10] J. Crisostomo, R. Troncoso and J. Zanelli, Phys. Rev. D 62, 084013 (2000). 
[11] A. H. Chamseddine, Phys. Lett. B 233, 291 (1989).

[12] R. G. Cai and K. S. Soh, Phys. Rev. D 59, 044013 (1999).

[13] R. Aros, R. Troncoso and J. Zanelli, Phys. Rev. D 63, 084015 (2001).

[14] M. Banados, C. Teitelboim and J. Zanelli, Phys. Rev. D 49, 975 (1994).

[15] G. Dotti, J. Oliva and R. Troncoso, Phys. Rev. D 75, 024002 (2007).

[16] P. Candelas and X. C. de la Ossa, Nucl. Phys. B 342, 246 (1990).

[17] P. Mora, R. Olea, R. Troncoso and J. Zanelli, JHEP 0406, 036 (2004).

[18] J. Lee and T. Parker, Bull. A. Math. Soc. (New Series), 17 (1987), 37.

[19] O. Miskovic, R. Troncoso and J. Zanelli, Phys. Lett. B 615, 277 (2005).

[20] C. Charmousis and J. F. Dufaux, Class. Quant. Grav. 19, 4671 (2002).

[21] R. Zegers, J. Math. Phys. 46, 072502 (2005).

[22] S. Deser and J. Franklin, Class. Quant. Grav. 22, L103 (2005).

[23] A. N. Aliev, H. Cebeci and T. Dereli, Class. Quant. Grav. 24, 3425 (2007).

[24] O. Aharony, S. S. Gubser, J. M. Maldacena, H. Ooguri and Y. Oz, Phys. Rept. 323, 183 (2000).

[25] E. Witten and S. T. Yau, Adv. Theor. Math. Phys. 3, 1635 (1999).

[26] J. M. Maldacena and L. Maoz, JHEP 0402, 053 (2004).

[27] N. Arkani-Hamed, J. Orgera and J. Polchinski, "Euclidean Wormholes in String Theory,"arXiv:0705.2768 [hep-th].

[28] A. H. Chamseddine, Nucl. Phys. B 346, 213 (1990).

[29] R. Troncoso and J. Zanelli, Int. J. Theor. Phys. 38, 1181 (1999).

[30] R. Aros, C. Martinez, R. Troncoso and J. Zanelli, JHEP 0205, 020 (2002).

[31] O. Miskovic, R. Troncoso and J. Zanelli, Phys. Lett. B 637, 317 (2006).

[32] G. Giribet, J. Oliva and R. Troncoso, JHEP 0605, 007 (2006).

[33] M. H. Dehghani, N. Bostani and A. Sheikhi, Phys. Rev. D 73, 104013 (2006).

[34] F. Mueller-Hoissen, Phys. Lett. B 163, 106 (1985).

[35] R. Aros, M. Romo and N. Zamorano, "Compactification in first order gravity," arXiv:0705.1162 [hep-th].

[36] R. G. Cai and N. Ohta, Phys. Rev. D 74, 064001 (2006).

[37] T. Torii and H. Maeda, Phys. Rev. D 71, 124002 (2005).

[38] G. Dotti and R. J. Gleiser, Class. Quant. Grav. 22, L1 (2005). 
[39] I. P. Neupane, Phys. Rev. D 69, 084011 (2004).

[40] R. J. Gleiser and G. Dotti, Phys. Rev. D 72, 124002 (2005).

[41] G. Dotti and R. J. Gleiser, Phys. Rev. D 72, 044018 (2005).

[42] M. Beroiz, G. Dotti and R. J. Gleiser, Phys. Rev. D 76, 024012 (2007)

[43] M. H. Dehghani and R. B. Mann, Phys. Rev. D 72, 124006 (2005).

[44] B. Bhawal and S. Kar, Phys. Rev. D 46, 2464 (1992).

[45] M. Thibeault, C. Simeone and E. F. Eiroa, Gen. Rel. Grav. 38, 1593 (2006).

[46] E. Gravanis and S. Willison, Phys. Rev. D 75, 084025 (2007).

[47] F. S. N. Lobo, Phys. Rev. D 75, 064027 (2007).

[48] G. Dotti, J. Oliva and R. Troncoso, work in preparation. 